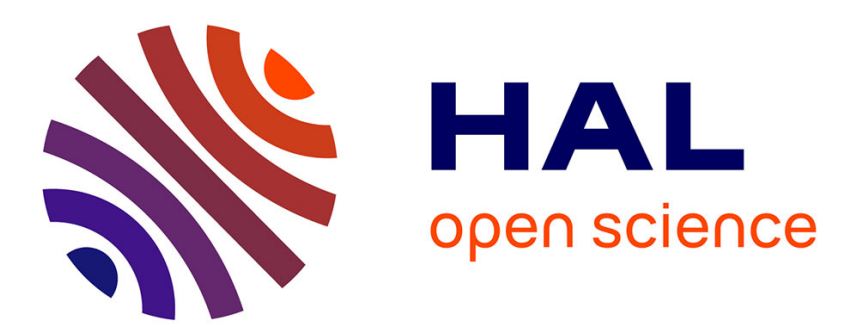

\title{
Perception de la parole et oscillations cérébrales chez les enfants neurotypiques et dysphasiques
}

Hélène Guiraud, Ana-Sofia Hincapié, Karim Jerbi, Véronique Boulenger

\section{To cite this version:}

Hélène Guiraud, Ana-Sofia Hincapié, Karim Jerbi, Véronique Boulenger. Perception de la parole et oscillations cérébrales chez les enfants neurotypiques et dysphasiques. 32èmes Journées d'Études sur la Parole, Jun 2018, Aix en Provence, France. 10.21437/JEP.2018-26 . hal-02460398

\section{HAL Id: hal-02460398 \\ https://hal.science/hal-02460398}

Submitted on 30 Jan 2020

HAL is a multi-disciplinary open access archive for the deposit and dissemination of scientific research documents, whether they are published or not. The documents may come from teaching and research institutions in France or abroad, or from public or private research centers.
L'archive ouverte pluridisciplinaire HAL, est destinée au dépôt et à la diffusion de documents scientifiques de niveau recherche, publiés ou non, émanant des établissements d'enseignement et de recherche français ou étrangers, des laboratoires publics ou privés. 


\title{
Perception de la parole et oscillations cérébrales chez les enfants neurotypiques et dysphasiques
}

\author{
Hélène Guiraud $^{1}$, Ana-Sofia Hincapié ${ }^{3}$, Karim Jerbi ${ }^{2}$ and Véronique Boulenger ${ }^{1}$
}

(1) Laboratoire Dynamique Du Langage, CNRS/Université Lyon 2 UMR5596, 14 avenue

Berthelot, 69007 Lyon, France

(2) Département de Psychologie, Université de Montréal, 90 avenue Vincent d'Indy, Montréal QC H2V2S9, Québec, Canada

(3) Pontificia Universidad Catolica de Chile, Av Libertador Bernardo O'Higgins, Santiago de Chile, Chile

guiraudh@gmail.com, ana.hincapie@gmail.com, karim.jerbi@umontreal.ca, veronique.boulenger@cnrs.fr

\section{RESUME}

L'hypothèse "prosodic phrasing" (Cumming et al., 2015) suggère que les enfants dysphasiques présentent des difficultés d'extraction des informations rythmiques de basse fréquence de la parole, entravant la segmentation syllabique et conduisant à des déficits phonologiques et morphosyntaxiques. Nous avons testé cette hypothèse en mesurant, en magnétoencéphalographie, la synchronisation entre les oscillations cérébrales et l'enveloppe temporelle du signal de parole chez des enfants dysphasiques et des enfants neurotypiques lors de l'écoute de phrases produites naturellement à un débit normal ou rapide. Nos résultats dans la bande de fréquence thêta $(4-7 \mathrm{~Hz})$ montrent une synchronisation plus faible chez les enfants dysphasiques, comparés aux enfants neurotypiques, 1) dans les régions auditives droites pour la parole à débit normal et 2) dans les régions (pré)motrices gauches pour la parole à débit rapide. Notre étude fournit les premiers éléments à notre connaissance en faveur d'un alignement cortical atypique sur le rythme syllabique dans la dysphasie.

\section{ABSTRACT}

According to the "prosodic phrasing" hypothesis (Cumming et al., 2015), children with Developmental Language Disorder (DLD) show difficulty extracting low-frequency rhythmic information from the speech signal, hindering syllabic segmentation and leading to phonological and morpho-syntactic impairments. We tested this hypothesis by measuring, using magnetoencephalography, the synchronization between cortical oscillations and speech amplitude envelope in children with DLD paired to typically-developing children when listening to sentences naturally produced at a normal or rapid rate. Our results in the theta frequency band $(4-7 \mathrm{~Hz})$ show reduced brain-to-speech coupling in children with DLD, as compared with typically-developing children, 1) in the right auditory cortex at normal rate and 2) left (pre)motor regions at fast rate. To our knowledge, this study brings the first piece of evidence for atypical cortical alignment to speech syllabic rhythm in children with DLD.

MOTS-CLÉS : parole, oscillations cérébrales, développement typique, dysphasie, débit KEYWORDS: speech, brain oscillations, typical development, Developmental Language Disorder, speech rate 


\section{Introduction}

La dysphasie est un trouble neurodéveloppemental sévère et durable de l'élaboration du langage oral qui ne peut être attribué à un trouble auditif, cognitif ou neurologique, un trouble du spectre autistique ou une carence affective ou éducative (Maillart, Schelstraete, 2012). La dysphasie peut affecter l'ensemble des composantes langagières de l'expression et/ou de la compréhension avec une sévérité variable ; les difficultés sont néanmoins le plus souvent décrites sur le plan phonologique et morphosyntaxique (i.e. dysphasie phonologico-syntaxique). Pour rendre compte de ces déficits, Cumming et collaborateurs (2015) ont avancé l'hypothèse de "prosodic phrasing " selon laquelle les enfants dysphasiques présenteraient une sensibilité réduite aux informations rythmiques contenues dans l'enveloppe temporelle de la parole, dont les modulations d'amplitude dominent dans les basses fréquences $(4-7 \mathrm{~Hz}$, thêta) et reflètent le débit syllabique du locuteur (Ghitza, Greenberg, 2009). Cette insensibilité entraverait alors la segmentation du signal en syllabes et en mots, et par conséquent le traitement de la structure phonologique et morphosyntaxique de la parole. Des études ont révélé que deux indices suprasegmentaux importants pour le traitement du rythme et de l'accentuation de la parole, le rise time (temps de montée des modulations successives de l'enveloppe d'amplitude) et la durée du signal, étaient particulièrement difficiles à traiter pour les enfants dysphasiques; ces difficultés étaient en outre corrélées à leurs performances phonologiques (Corriveau et al., 2007; Cumming et al., 2015). Nous avons par ailleurs montré des performances réduites chez les enfants dysphasiques, en regard d'enfants au développement typique (dits neurotypiques), pour comprendre la parole accélérée naturellement ou artificiellement, suggérant un déficit de traitement du rythme de la parole dès lors qu'il est accéléré (Guiraud et al., sous presse).

Soulignant la correspondance étroite entre le rythme de la parole et les rythmes intrinsèques du cerveau, les modèles neurocognitifs actuels suggèrent que les oscillations corticales jouent un rôle majeur dans le décodage du signal verbal (Ghitza, 2011 ; Giraud, Poeppel, 2012). En particulier, les oscillations du cortex auditif dans la bande de fréquence thêta $(4-7 \mathrm{~Hz})$ seraient capables de se synchroniser sur l'enveloppe temporelle de la parole, permettant au cerveau de l'auditeur de segmenter le flux continu en unités pertinentes, les syllabes, et d'ainsi faciliter le traitement linguistique. Il a été suggéré que les déficits de traitement rythmique dans les troubles développementaux du langage puissent résulter d'une synchronisation atypique des oscillations corticales sur le signal de parole, en particulier dans les basses fréquences (Giraud, Poeppel, 2012 ; Goswami, 2011). Bien que l'hypothèse d'un dysfonctionnement cortical oscillatoire dans la dysphasie soit particulièrement séduisante, elle n'a jamais fait l'objet d'investigations à l'heure actuelle et à notre connaissance. Des travaux ont néanmoins révélé un fonctionnement oscillatoire atypique dans la dyslexie, caractérisé par un trouble de la synchronisation des oscillations corticales sur le signal de parole dans les basses et hautes fréquences (e.g., Molinaro et al., 2016).

Dans cette étude, nous avons examiné, à l'aide d'une technique d'imagerie cérébrale non invasive, la magnétoencéphalographie (MEG), la dynamique des oscillations cérébrales lors de la perception de parole chez des enfants dysphasiques et des enfants neurotypiques. Nous nous sommes intéressés au cas de la parole produite naturellement à un débit normal ou rapide, afin de déterminer dans quelle mesure les oscillations corticales étaient capables de "suivre" le rythme syllabique. Notre hypothèse était que chez les enfants neurotypiques, les régions auditives devraient synchroniser leur activité oscillatoire thêta $(4-7 \mathrm{~Hz})$ sur la parole dans les deux conditions de débit, avec l'implication 
éventuelle de régions supplémentaires pour traiter la parole rapide, plus difficile à comprendre. Chez les enfants dysphasiques, en accord avec le modèle de Cumming et al. (2015) et notre étude comportementale (Guiraud et al., sous presse), nous nous attendions à une synchronisation thêta atypique (i.e. couplage réduit et/ou réseaux corticaux différents) entre rythmes cérébraux et linguistiques, comparés aux enfants neurotypiques, pour les deux conditions de débit.

\section{Matériel et méthode}

\subsection{Participants}

Onze enfants présentant une dysphasie phonologico-syntaxique (DYS, âge moyen 10.29 ans, EcartType OU ET 1.54), appariés en âge et sexe à 11 enfants neurotypiques (NT, âge moyen 10.83 ans, ET 1.65), ont participé à l'étude. Tous les enfants étaient âgés de 8 à 13 ans, de langue maternelle française (non bilingues), droitiers et sans trouble de l'audition (vérification par audiométrie tonale). Les enfants DYS respectaient différents critères d'inclusion (QI non-verbal $>70$ et troubles prédominants sur le versant expressif avec une compréhension préservée) et d'exclusion (déficit de l'attention/hyperactivité, trouble du spectre autistique, retard intellectuel). Avant l'expérience, les capacités verbales (phonologie, morphosyntaxe, compréhension, vocabulaire) de tous les enfants ont été évaluées grâce à différents sous-tests de la BALE (Batterie Analytique du Langage Écrit) et de l'ELO (Évaluation du Langage Oral). Leurs capacités non-verbales ont été examinées à l'aide des matrices progressives colorées de Raven et de l'empan endroit et envers de chiffres. Enfin, le débit de parole des enfants a été mesuré dans une tâche narrative de description d'images ("Frog where are you?") : il était de $1.81 \mathrm{syll} / \mathrm{s}$ (ET 0.43 ) chez les DYS et de $2.45 \mathrm{syll} / \mathrm{s}$ (ET 0.70) chez les NT ( $p$ $=.02$ ). Le protocole était conforme à la déclaration d'Helsinki et a reçu un avis favorable du Comité de Protection des Personnes (ID RCB: 2012-A00857-36). Tous les enfants et leurs parents ont signé un formulaire de consentement éclairé avant l'expérience.

\subsection{Stimuli}

Trois cents phrases (7-9 mots) de structure syntaxique identique (e.g., "Le public applaudit le joueur pour sa victoire") ont été enregistrées $(44.1 \mathrm{kHz}$, mono, 16 bits) par un locuteur français de sexe masculin dans une salle insonorisée à l'aide du logiciel ROCme!. Chaque phrase a été enregistrée à un débit normal (moyenne $6.61 \mathrm{syll} / \mathrm{s}$, ET 0.47) puis rapide (moyenne $9.03 \mathrm{syll} / \mathrm{s}$, ET 0.56). Un filtre passe-haut de $80 \mathrm{~Hz}$ ainsi qu'un fondu d'entrée et de sortie sur l'enveloppe d'amplitude ont été appliqués sur la totalité des fichiers sons avec le logiciel Praat; le pic d'intensité a également été normalisé. Les 600 phrases $(2 \times 300)$ ont été réparties dans deux listes expérimentales de 300 stimuli (150 à débit normal, 150 à débit rapide). La fréquence d'occurrence dans la langue des mots finaux des phrases, ainsi que leurs nombres de phonèmes et de voisins phonologiques, ne différaient pas significativement entre les listes. Chaque phrase apparaissait dans chaque condition de débit à travers tous les participants, mais une seule fois par liste. Quarante phrases «fillers » (20 de chaque débit, non analysées), similaires aux phrases cibles mais à la fin desquelles un son était ajouté (http://www.sound-fishing.net/), ont été créées et ajoutées à chaque liste expérimentale pour un total 
de 340 stimuli par liste. Au sein de chaque liste, les stimuli étaient pseudo-randomisés (pas plus de 3 phrases consécutives de la même condition). Afin d'analyser le couplage entre le signal de parole et l'activité cérébrale oscillatoire, l'enveloppe d'amplitude de chaque phrase a été extraite selon la méthode de Peelle et al. (2013 ; signal rectifié et filtré à $30 \mathrm{~Hz}$ avec un filtre passe-bas). Cette enveloppe a constitué le signal acoustique utilisé dans les analyses présentées en 2.4.

\subsection{Procédure}

Les enfants étaient confortablement allongés dans une chambre d'enregistrement insonorisée et blindée, la tête positionnée dans le casque MEG (système "tête entière » à 275 canaux, OMEGA MEG CTF 275 ; échantillonnage à $1200 \mathrm{~Hz}$ ). Ils étaient équipés d'écouteurs intra-auriculaires et un écran était placé à $40 \mathrm{~cm}$ devant eux. Avant de débuter l'enregistrement MEG, un seuil auditif était réalisé pour chaque oreille afin de vérifier que tous les enfants entendaient les stimuli à la même intensité (détection d'un son pur de 400 à $3000 \mathrm{~Hz}$ ). L'intensité était ensuite ajustée de sorte que les stimuli verbaux soient présentés en stéréo à $50 \mathrm{~dB}$ SL. Au cours de l'enregistrement, les enfants avaient pour consigne d'écouter attentivement les phrases (en fixant une croix sur l'écran) et d'appuyer sur un bouton avec leur index gauche lorsqu'ils entendaient un stimulus filler. Cinq phrases d'entrainement étaient proposées avant le début de la tâche. L'expérience a été réalisée avec le logiciel Présentation (Neurobehavioral Systems).

\subsection{Analyses MEG}

Les analyses ont été réalisées avec Matlab (Mathworks Inc., MA, USA) et la toolbox Fieldtrip. Après avoir ré-échantillonné les données à $300 \mathrm{~Hz}$ et supprimé les artéfacts musculaires, oculaires et cardiaques, des analyses de sources ont été réalisées. Pour cela, un modèle (template) de cerveau enfant (pour la tranche d'âge 7.5-13.5 ans), aligné sur les points fiduciaux (nasion et points préauriculaires) des participants de notre expérience, a été utilisé afin de créer un modèle de tête individuel pour chaque enfant. Ces modèles individuels ont ensuite été transposés sur un template adulte afin d'effectuer les analyses de sources dans un système de coordonnées commun (Montreal Neurological Institute ou MNI). Ceci nous a permis d'utiliser un atlas anatomique adulte pour les analyses statistiques basées sur des régions d'intérêt (ROIs). Nous avons mesuré la cohérence entre le signal MEG et l'enveloppe d'amplitude du signal de parole (i.e. cohérence cortico-acoustique) ainsi que les modulations de puissance au niveau des sources corticales à l'aide d'un filtre spatial (DICS). La cohérence correspond ici à la relation linéaire entre l'amplitude de deux signaux dans le domaine fréquentiel. Pour chaque essai, nous avons défini une période active $(0.2$ à $1 \mathrm{~s}$ après le début de la phrase) et une période de référence (intervalle inter-stimulus, $-0.2 \mathrm{à}-1 \mathrm{~s}$ avant la phrase). La cohérence cortico-acoustique a été calculée pour toutes les périodes actives et de référence pour les deux conditions de débit dans la bande de fréquence thêta $(4-7 \mathrm{~Hz})$ qui englobe les fluctuations lentes d'amplitude de l'enveloppe temporelle de la parole. Les moyennes de chaque groupe (DYS et NT) dans chaque condition de débit ont été calculées, et des statistiques non paramétriques avec la méthode de Monte-Carlo (1000 répétitions, correction au niveau des clusters) ont été réalisées sur 4 ROIs bilatérales définies selon les modèles de la parole (Giraud, Poeppel, 2012; Hickok, Poeppel, 2007) : gyrus de Heschl, gyrus temporal supérieur, gyrus temporal médian et cortex précentral (atlas Automated Anatomical Labeling ou AAL). Nous avons également conduit des analyses de 
corrélation de Spearman entre la cohérence cortico-acoustique au sein des ROIs et les performances langagières des enfants DYS et NT telles que mesurées par la BALE et l'ELO (cf. partie 2.1).

\section{Résultats}

La FigURE 1 présente les cartes de cohérence cortico-acoustique dans la bande thêta (4-7 Hz) chez les enfants dysphasiques (1A) et les enfants neurotypiques (1B) dans les conditions de débit normal et de débit rapide, ainsi que le contraste direct entre les deux groupes (1C). Lors de la perception de parole à débit normal, chez les enfants NT, une augmentation de cohérence entre le signal cortical MEG et l'enveloppe d'amplitude des phrases est principalement observée dans le cortex temporal médian et supérieur antérieur droit ainsi que dans le cortex fronto-pariétal droit. Chez les enfants DYS en revanche, ce pattern n'est pas retrouvé. Les analyses statistiques montrent en effet un couplage cortico-acoustique réduit chez ces enfants, comparés à leurs pairs au développement typique, au sein des gyri temporaux médian et supérieur antérieurs droits (Aires de Brodmann BA 21 et $22 ; 1 \mathrm{D}$, panneau de gauche) en condition de débit normal. Notons que ceci est observé de manière bilatérale lorsque la correction statistique n'est pas appliquée (analyses non corrigées, 1E, panneau de gauche). Lors de la perception de parole rapide, aucune différence significative entre les groupes n'est observée pour les analyses corrigées. Cependant, les résultats non corrigés (1E, panneau de droite) révèlent un alignement plus fort des oscillations thêta sur le signal acoustique dans le cortex prémoteur gauche (BA 6 et 8), la partie antérieure du cortex temporal supérieur droit (BA 22) et le cortex auditif primaire droit (BA 41) chez les enfants NT comparés aux enfants DYS. Les enfants DYS montrent quant à eux une augmentation de cohérence au niveau du cortex temporal supérieur postérieur droit (BA 22) et des gyri angulaire et supramarginal droits (BA 39 et 40) dans cette condition de débit, alors qu'elle n'est pas observée chez les enfants NT.

Il faut par ailleurs noter que les analyses des puissances dans la bande thêta n'ont montré aucune modulation significative (corrigée ou non) de la puissance dans les régions corticales précitées lors de la perception de parole à débit normal ou rapide (par rapport à la période de référence), chez aucun des deux groupes d'enfants. Ceci suggère que les augmentations de cohérence corticoacoustique observées reflètent bien un processus de synchronisation oscillatoire et non simplement une augmentation de l'activité corticale. 


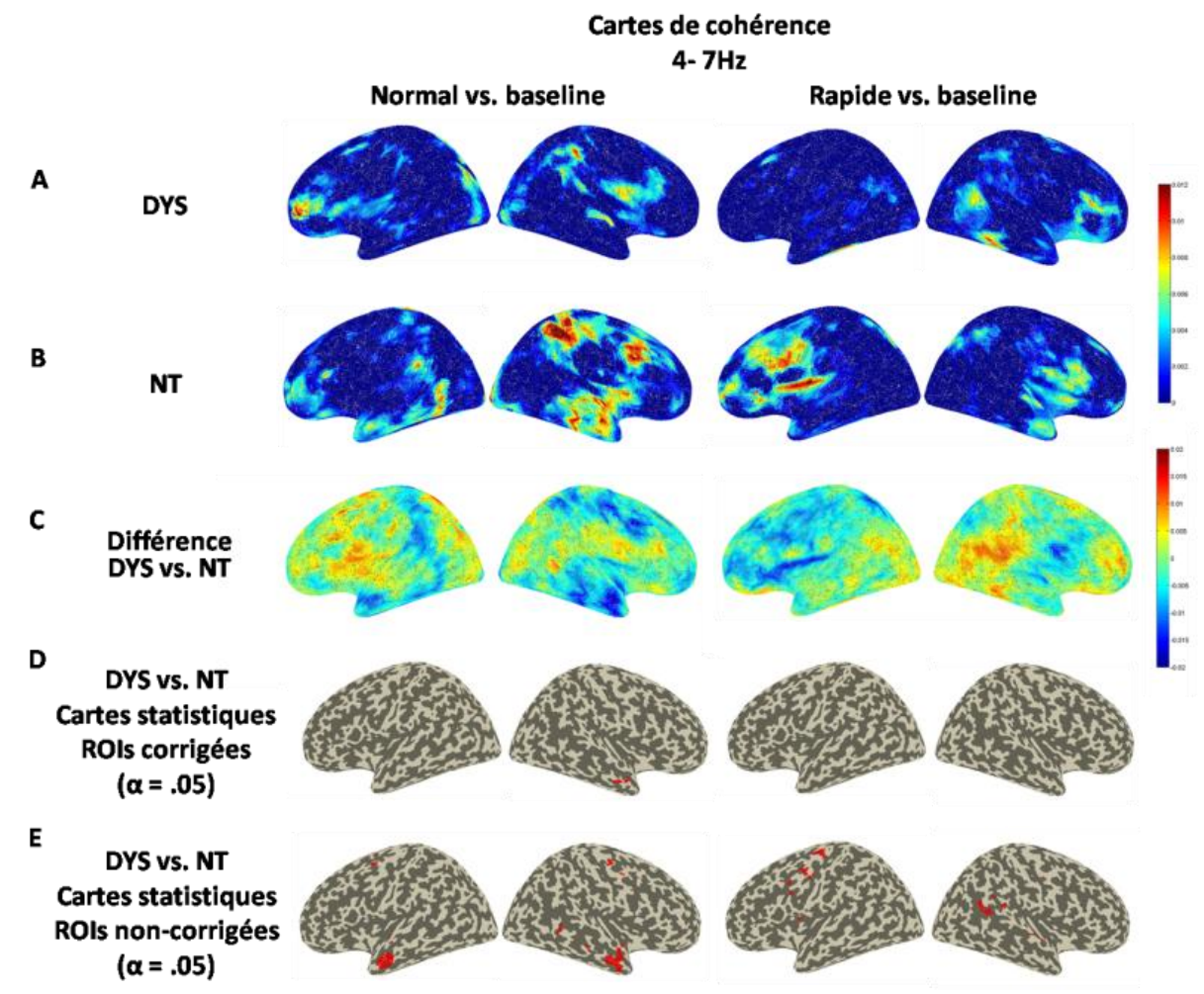

FIGURE 1 : Cartes de cohérence cortico-acoustique dans la bande thêta (4-7 Hz) lors de la perception de parole à débit normal (panneau de gauche) et à débit rapide (panneau de droite), par rapport à la ligne de base (période de référence ou baseline), chez les enfants dysphasiques (A) et les enfants neurotypiques (B). Le contraste direct entre les deux groupes est présenté en C. Les cartes statistiques corrigées (D) et non corrigées (E) de cette différence sont également reportées.

Les analyses de corrélation entre la cohérence cortico-acoustique dans la bande thêta (4-7 Hz) et les performances langagières des enfants ne montrent aucune corrélation significative chez les enfants NT. En revanche, chez les enfants DYS, elles révèlent d'une part une corrélation négative entre la cohérence dans la région temporale médiane postérieure droite (incluant le gyrus angulaire) et les performances phonologiques des enfants (tâches de répétition et métaphonologiques ; $\mathrm{r}=-0.75, \mathrm{p}<$ .01, Figure 2A). D'autre part, une corrélation positive est observée entre la cohérence dans le cortex temporal supérieur postérieur droit (englobant le gyrus supramarginal) et le propre débit de parole des enfants mesuré dans une tâche narrative $(\mathrm{r}=0.91, p<.001$, FIGURE 2B). Autrement dit, plus les enfants DYS sont capables de parler vite, plus le cortex temporal postérieur droit synchronise son activité oscillatoire sur l'enveloppe d'amplitude des signaux de parole rapide. Ce couplage cortico-acoustique est par ailleurs d'autant plus présent que les enfants ont des performances phonologiques faibles. Dans l'ensemble, ces corrélations suggèrent donc que l'augmentation de cohérence dans le cortex temporal postérieur droit chez les enfants dysphasiques reflète un pattern atypique et la mise en place possible d'un phénomène compensatoire pour traiter la parole rapide. 

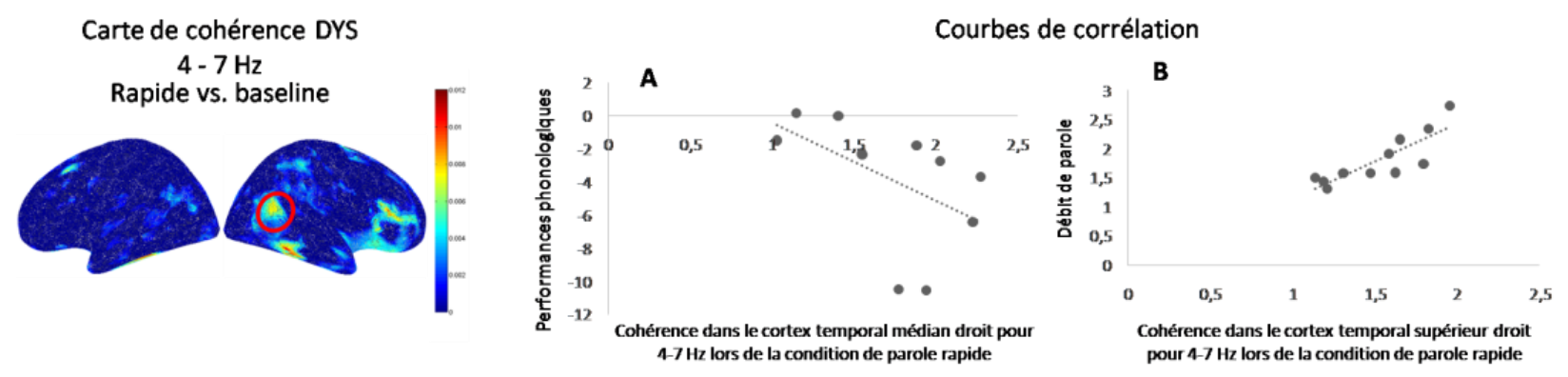

FIGURE 2 : Corrélations de Spearman (rho) entre (A) la valeur moyenne de cohérence corticoacoustique dans la bande thêta $(4-7 \mathrm{~Hz})$ dans le cortex temporal médian postérieur droit lors de la perception de parole rapide et les performances phonologiques des enfants DYS, et (B) la valeur moyenne de cohérence en 4-7 Hz dans le cortex temporal supérieur droit dans la condition rapide et le propre débit de parole des enfants. Les valeurs sont exprimées en scores $Z$.

\section{Discussion}

Les résultats dans la condition de parole à débit normal suggèrent un alignement plus faible des oscillations corticales thêta dans le cortex auditif associatif droit sur l'enveloppe d'amplitude du signal chez les enfants dysphasiques, comparés à des enfants neurotypiques du même âge. Nos observations chez les enfants neurotypiques s'accordent avec les modèles oscillatoires ainsi qu'avec les études chez l'adulte (Giraud, Poeppel, 2012) montrant un couplage cortico-acoustique dans la bande thêta préférentiellement dans le cortex auditif droit, qui serait plus à même de segmenter la parole en unités syllabiques. Les rares études menées chez l'enfant, utilisant des stimuli non verbaux ou verbaux très simples, ont également décrit ce pattern de latéralisation (Abrams et al., 2008). Notre étude révèle qu'alors que les enfants neurotypiques âgés de 8 à 13 ans synchronisent leurs oscillations corticales sur le rythme syllabique de phrases naturellement produites à un débit normal, ceci ne semble pas être le cas chez les enfants dysphasiques. Les régions auditives droites des dysphasiques seraient ainsi moins capables de suivre les fluctuations lentes de l'enveloppe d'amplitude de la parole, même produite à un débit normal, ce qui conduirait à une extraction moins efficace de l'information rythmique (syllabique ici) et par conséquent au développement de représentations phonologiques et morphosyntaxiques imprécises. Dans l'ensemble, nos données s'accordent donc avec les prédictions de la «prosodic phrasing hypothesis » (Cumming et al., 2015) et les études montrant l'existence de déficits de traitement du rythme chez les dysphasiques (Corriveau et al., 2007).

Lorsque la parole est accélérée naturellement, des réseaux corticaux distincts et présentant une latéralisation hémisphérique différente semblent être engagés chez les deux groupes d'enfants (rappelons que les résultats étaient significatifs sans correction statistique et mériteraient donc d'être confirmés avec un plus grand échantillon). Alors que les régions (pré)motrices gauches se synchronisent sur la parole rapide chez les enfants neurotypiques, le couplage cortico-acoustique est observé dans le cortex temporal postérieur droit chez les enfants dysphasiques. Les travaux chez l'adulte montrent une implication spécifique des régions motrices gauches ainsi qu'une connectivité fonctionnelle renforcée entre les régions auditives et motrices lors de la perception de parole dans des conditions difficiles (Alho et al., 2014). Nos données chez l'enfant neurotypique révèlent que 
lorsque la parole est produite plus rapidement (i.e. plus difficile à décoder), l'activité oscillatoire des régions de production de la parole se synchronise sur les modulations d'enveloppe du signal. Ceci est en accord avec l'existence d'une voie dorsale d'intégration sensori-motrice (modèle à double voie ; Hickok, Poeppel, 2007) ainsi qu'avec les études suggérant un rôle prédictif des régions articulatoires dans le traitement de l'information auditive temporelle (Morillon et al., 2014). Chez les enfants dysphasiques, nous n'avons observé aucun couplage cortico-acoustique dans le cortex (pré)moteur gauche, évoquant un dysfonctionnement possible de la voie dorsale sensori-motrice lorsqu'il s'agit de s'aligner sur un rythme syllabique rapide. Ceci pourrait rendre compte du déficit de perception de parole rapide que nous avions précédemment mis en évidence chez un autre groupe d'enfants dysphasiques (Guiraud et al., 2018). Nos résultats s'accordent également avec les travaux montrant que les troubles rythmiques dans la dysphasie s'expriment non seulement au niveau perceptif mais aussi moteur (i.e. tâches de tapping) et qu'ils pourraient donc refléter un dysfonctionnement du couplage auditivo-moteur (Corriveau, Goswami, 2009). Par ailleurs, chez les dysphasiques, nous avons mis en évidence une synchronisation des oscillations du cortex temporal postérieur droit, s'étendant à la jonction temporo-pariétale, sur la parole rapide, qui n'était pas retrouvée chez les enfants neurotypiques. Cette région appartient à la voie dorsale sensori-motrice, normalement latéralisée à gauche, et est censée participer aux processus phonologiques à la fois en perception et en production. Il est notamment intéressant de noter que des études neuroanatomiques et fonctionnelles ont révélé une absence de latéralisation à gauche (observée dans le développement typique), voire une latéralisation à droite, de cette région chez les dysphasiques (Badcock et al., 2012). Nos résultats, s'ils sont confirmés avec un plus grand échantillon, pourraient alors suggérer que l'implication de la région temporale postérieure droite lors de la perception de parole rapide chez les enfants dysphasiques reflète un phénomène compensatoire. La voie dorsale sensori-motrice pourrait être fonctionnelle mais présenterait une latéralisation hémisphérique différente de celle observée chez les enfants neurotypiques (voir Cutini et al., 2016 chez des enfants dyslexiques). Cette interprétation semble corroborée par les résultats préliminaires de nos analyses de corrélation : si l'activité du cortex temporal postérieur droit reflète des mécanismes d'intégration sensori-motrice, la plus forte synchronisation oscillatoire dans cette région sur la parole rapide chez les enfants dysphasiques capables de parler vite pourrait indiquer que le couplage auditivo-moteur (même latéralisé à droite) se produise dans une certaine mesure. Autrement dit, la région temporale droite d'intégration sensori-motrice serait plus à même de décoder la parole rapide chez les enfants dysphasiques présentant moins de troubles expressifs. Néanmoins, l'implication de cette région droite était particulièrement observée chez les enfants présentant des troubles phonologiques sévères (corrélation négative). Bien qu'aucune relation causale ne puisse être inférée à partir de nos analyses actuelles, ceci suggère que nos résultats reflètent un pattern atypique, en termes d'asymétrie hémisphérique notamment, du traitement de la parole dans la dysphasie.

En conclusion, notre étude apporte, pour la première fois à notre connaissance, des éléments en faveur d'un alignement atypique entre rythme syllabique et rythmes cérébraux lors de l'écoute de parole naturelle à débit normal et rapide chez les enfants dysphasiques.

\section{Remerciements}

ANR ODYSSEE (PI : V.B., n¹1 JSH2 005 1) ; LabEx ASLAN (ANR-10-LABX-0081, bourse de doctorat: H.G.) et Erasmus Mundus Auditory Cognitive Neuroscience (H.G.) ; Programme des Chaires du Canada et de la Subvention à la découverte du CRSNG (K.J. ; RGPIN-2015-04854). 


\section{Références}

ABRAMS D., NICOL T., ZECKER S., KRAUS N. (2008). Right-hemisphere auditory cortex is dominant for coding syllable patterns in speech. Journal of Neuroscience 28(15), 3958-3965.

ALHO J., LIN F.-H., SATO M., TIITINEN H., SAMS M., JÂÂSKELÄINEN I. P. (2014). Enhanced neural synchrony between left auditory and premotor cortex is associated with successful phonetic categorization. Frontiers in Psychology 5, 394.

BADCOCK N. A., BISHOP D. V. M., HARDIMAN M. J., BARRY J. G., WATKINS K. E. (2012). Colocalisation of abnormal brain structure and function in specific language impairment. Brain and Language $120(3), 310-320$.

CORRIVEAU K. H., PASQUINI E. S., GOSWAMI U. (2007). Basic auditory processing skills and specific language impairment: a new look at an old hypothesis. Journal of Speech Language and Hearing Research 50(3), 647-666.

CUTINI, S., SZUCS, D., MEAD, N., HUSS, M., GOSWAMI, U. (2016). Atypical right hemisphere response to slow temporal modulations in children with developmental dyslexia. NeuroImage, 143, 40-49.

CORRIVEAU K. H., GOSWAMI U. (2009). Rhythmic motor entrainment in children with speech and language impairments: tapping to the beat. Cortex 45, 119-130.

CUMMING R., WiILSON A., GOSWAMI U. (2015). Basic auditory processing and sensitivity to prosodic structure in children with specific language impairments: a new look at a perceptual hypothesis. Frontiers in Psychology 6, 972.

GHITZA O. (2011). Linking speech perception and neurophysiology: speech decoding guided by cascaded oscillators locked to the input rhythm. Frontiers in Psychology 2, 130.

GHITZA O., GREENBERG S. (2009). On the possible role of brain rhythms in speech perception: intelligibility of time-compressed speech with periodic and aperiodic insertions of silence. Phonetica 66(1-2), 113-126.

GIRAUD A.-L., POEPPEL, D. (2012). Cortical oscillations and speech processing: emerging computational principles and operations. Nature Neuroscience 15(4), 511-517.

GUIRAUD H., BEDOIN N., KRIFI-PAPOZ S., HERBILLON V., CAILLOT-BASCOUL A., GONZALEZMONGE S., BOULENGER V. (sous presse). Don't speak too fast! Processing of fast rate speech in children with Specific Language Impairment. PLOS ONE.

HICKOK G., POEPPEL D. (2007). The cortical organization of speech processing. Nature Reviews Neuroscience 8(5), 393-402.

MAILLART C., SCHELSTRAETE M. A. (2012). Les dysphasies: de l'évaluation à la rééducation. Issy-lesMoulineaux, France : Elsevier-Masson.

MOLINARO N., LIZARAZU M., LALLIER M., BOURGUIGNON M., CARREIRAS M. (2016). Out-ofsynchrony speech entrainment in developmental dyslexia. Human Brain Mapping 37(8), 2767-2783.

MORILLON B., SCHROEDER C. E., WYART V. (2014). Motor contributions to the temporal precision of auditory attention. Nature Communications 5, 1-9.

PEELLE J. E., GROSS J., DAVIS, M. H. (2013). Phase-Locked Responses to Speech in Human Auditory Cortex are Enhanced During Comprehension. Cerebral Cortex 23(6), 1378-1387. 